\title{
Metacognición institucional para un aprendizaje organizacional participativo: Herramienta innovadora de gestión escolar
}

\author{
Organizational Metacognition to participatory learning in organizations: Innovative tool for school \\ management
}

Recibido 12 junio 2014 • Aceptado 26 setiembre 2015 • Corregido 05 octubre 2015

\author{
Carlos Javier Ossa Cornejo' \\ Universidad del Bío-Bío, Chile \\ cossa@ubiobio.cl \\ Fancy Inés Castro Rubilar ${ }^{2}$ \\ Universidad del Bío-Bío, Chile \\ fcastro@ubiobio.cl \\ María Teresa Castañeda Díaz \\ Universidad del Bío-Bío, Chile \\ mcastane@ubiobio.cl \\ Juana Irene Castro Rubilar ${ }^{4}$ \\ Universidad del Bío-Bío, Chile \\ icastro@ubiobio.cl
}

1 Doctor $\odot$ en Psicología, en la línea de enseñanza y aprendizaje, Universidad de Concepción, Chile. Magíster en Educación, Universidad Academia de Humanismo Cristiano, Chile. Psicólogo Educacional. Actualmente es académico de la Universidad del Bio-Bio, Chile, en formación de pregrado de carreras de pedagogía y en postgrado, en el Magíster en Educación y en el Magíster en Liderazgo y Gestión de Establecimientos Educativos, Universidad del Bío-Bío.

2 Doctora en Diseño Curricular y Evaluación Educacional, Universidad de Valladolid, España. Magíster en Educación, Mención Escolar, Universidad Metropolitana de Ciencias de la Educación, Chile. Profesora de Estado en Castellano, Universidad del Bío-Bío, Chile. Actualmente se desempeña como académica en la Facultad de Educación y Humanidades de la Universidad del Bío-Bío. Es Directora del Programa de Magíster en Liderazgo y Gestión de Establecimientos Educacionales de la misma institución.

3 Doctora $\odot$ en Educación, Universidad de la Frontera, Chile. Magíster en Currículum, Evaluación e Innovación Educativa. Profesora de Estado en Historia, Geografía y Educación Cívica. Actualmente es académica del Departamento de Ciencia de la Educación, Universidad del Bío-Bío.

4 Doctora en Investigación Educativa de la Universidad de Alicante, España. Magíster en Educación con mención en Currículum. Diplomada en Alta Dirección Pública, Universidad de Chile. Profesora de Educación General Básica de la Pontificia Universidad Católica de Chile. Actualmente se desempeña como académica de la Universidad del Bío-Bío. Representante de la Comisión de Alta Dirección Pública de Chile. 
Resumen. El siguiente ensayo analiza la metacognición en relación con las nuevas perspectivas constructivistas y como forma de aprendizaje asociado a la gestión del conocimiento y el aprendizaje organizativo. Desde esta nueva perspectiva, se valora el conocimiento de los sujetos no solo como una característica intraindividual, sino más bien interindividual (o grupal) y organizacional. Se presenta el concepto de metacognición institucional como proceso que permite generar un aprendizaje consciente, dialógico y participativo en educación. Esta mirada organizacional del conocimiento y la metacognición es relevante para el ámbito de gestión escolar, porque implica la necesidad de construir una nueva visión de la escuela como una organización que no solo enseña, sino que aprende $y$, en consecuencia, entrega herramientas novedosas y pertinentes a las actuales necesidades sociales de comunicación y participación que tensionan, hoy en día, a las organizaciones educativas.

Palabras clave. Metacognición institucional; aprendizaje; gestión participativa

\begin{abstract}
This essay discusses about metacognition related to new constructivist perspectives and as a form of learning associated with knowledge management and organizational learning. From this new perspective, the knowledge of the subject is valued not only as an intraindividual feature, but rather inter-individual (or group) and organizational. The concept of institutional metacognition as a process that generates a conscious, dialogical and participatory learning in education is presented. This organizational perspective of learning and metacognition is relevant to the field of school management, because it implies the need to build a new vision of the school as an organization that not only teaches but learns and therefore delivers new tools and relevant to current social needs of communication and participation that are stressed nowadays educational organizations.
\end{abstract}

Keywords. Institutional metacognition; learning; participatory management

\title{
Metacognición y aprendizaje: desde lo intraindividual a lo interindividual
}

La metacognición es un concepto que ha estado muy relacionado con el aprendizaje desde los modelos cognitivos elaborados en la década del 70; se ha visto como un proceso de pensamiento superior, relacionado con el pensamiento productivo y creativo (Arancibia, Herrera y Strasser, 2008), permite el desarrollo de ideas para mejorar el desempeño del pensamiento. Este concepto surge aproximadamente en el año 1976, desarrollado por Flavell (Mateos, 2001), quien lo define como el conocimiento que una persona posee acerca de los propios procesos y productos cognitivos. Si bien se ha conceptualizado tradicionalmente como una habilidad individual, existe hoy en día una variada literatura que señala su potencial como habilidad de trabajo grupal y organizacional (Zorzoli, Lopardo y Pérez, 1999; Briñol y De Marre, 2012; Larkin, 2009; Tovar, 2008) relacionada con estrategias de comunicación y trabajo en equipo.

Existen numerosos trabajos e investigaciones que hablan de la utilidad de la metacognición para el aprendizaje; por consiguiente, las capacidades metacognitivas que el alumno posee 
en mayor o menor grado, condicionan su aprovechamiento en las tareas de aprendizaje y se consideran mejores indicios de rendimiento académico que otras mediciones intelectuales (Akyol y Garrison, 2011; Añino y Perazzi, 2008; Larkin, 2009; Martínez Fernández, 2004; Mateos, 2001; Sandi-Urena, Cooper y Stevens, 2011; Tovar, 2008); asimismo, el tener acceso al conocimiento metacognitivo facilitaría la resolución de problemas al hacer consciente los procedimientos de razonamiento implicados en esa tarea (Pozo, 2001).

De este modo, se puede plantear que si una persona tiene conocimiento de sus propios procesos psicológicos, podrá usarlos más eficaz y flexiblemente en la planificación de sus estrategias de aprendizaje, que serían las secuencias de procedimientos y actividades cognitivas que se integran con el propósito de facilitar la adquisición, almacenamiento y/o empleo de la información. Es relevante considerar que este proceso metacognitivo se ha planteado como una declaración consciente del procesamiento de información, con una estructura basada tanto en procesos de conocimiento del aprendizaje como en los procesos de control del mismo, por lo que es una habilidad del pensamiento que permite conocer profundamente lo que pensamos y hacemos, y a la vez, controlar estos procedimientos.

Al analizar la forma en que los procesos metacognitivos permiten articular aspectos de la cognición del estudiante con la intervención didáctica del docente, se puede entrever un medio que aporta a que el estudiante sea autónomo en términos de aprender a aprender; y también, se puede vislumbrar un tratamiento en el que el docente reflexiona sobre sus conocimientos específicos de la disciplina académica, sus conocimientos pedagógico-didácticos y sobre sus epistemologías, consiguiendo aportar a su estudiante, consecuentemente, ideas para mejorar su aprendizaje, así como tener referentes claros para guiar su propia formación. Es por ello que, más que intraindividual, la generación de estrategias de aprendizaje que se basan en la metacognición son interindividuales (grupales).

De este modo, lo interindividual se estructura en una perspectiva constructivista, permitiendo rescatar características del pensamiento, olvidadas y negadas por la perspectiva simplista y reduccionista en que ha caído muchas veces la psicología y que guardan relación con el diálogo y la interacción. Se rescata una mirada del aprendizaje relacionada con el cambio activo de la información en el sujeto, que no puede más que generarse en una organización mediada y resignificadora con los integrantes de su medio, detectando los aciertos y errores a partir del diálogo (Larkin, 2009; Pozo, 2001).

La metacognición individual (intraindividual) estaría orientada a monitorear y controlar los propios procesos de conocimiento, emociones y acciones; mientras que la metacognición social (interindividual) consistiría en que los miembros del grupo ayudan a monitorearse unos a otros en sus procesos de conocimiento, emociones y acciones. Lo anterior, permitiría distribuir las responsabilidades entre los miembros del grupo, aumentando el aprendizaje y los procesos cognitivos (Akyol y Garrison, 2011; Chin y Kuo, 2010; Sandi-Urena et al., 2011). 
Los individuos no aprenderían por el hecho de que usen procesos cognitivos individuales, sino porque ejecutan algunas actividades que involucran ciertos mecanismos de aprendizaje en un entorno de colaboración y reciprocidad, el cual les permite darse cuenta de sus éxitos y errores. Del mismo modo, los pares no aprenden porque sean dos, sino porque ejecutan algunas actividades que conllevan mecanismos de aprendizaje específicos, que son ejecutados en un contexto de diálogo, el cual genera actividades cognitivas adicionales (Collazos y Mendoza, 2006).

\section{Aprendizaje organizacional y metacognición: herramientas de apoyo a la gestión escolar}

Se ha planteado en el acápite anterior que la metacognición serviría de herramienta para promover el aprendizaje interindividual, logrando que no solamente las personas individuales mejoren sus procesos de aprendizaje, sino también lo realicen grupos de personas en un trabajo colaborativo. Se desea señalar en este apartado, que este mejoramiento no solamente puede ser de grupos de personas, sino que además, podría llevar al mejoramiento de la organización en general, a través de la formación basada en reflexión metacognitiva que permitiría llevar a los integrantes de la organización a un estado de mayor conciencia de sus acciones y necesidades, estado al cual no podrían llegar fácilmente por sí solos, sino con el apoyo de un mediador que fomente el nivel de reflexión metacognitiva requerido.

Esto se enmarca en la idea de que la organización no solo procesa conocimiento, sino que además lo crea, entendiendo esto como la capacidad de una compañía en su conjunto para crear nuevo saber, diseminarlo a través de la organización y encarnarlo en productos, servicios y sistemas. De ese mismo modo, una institución que aprende a aprender, es una institución que se permite tener apoyos para lograr un cambio trascendente y significativo en sus pautas y acciones frente a las necesidades dinámicas de su comunidad educativa (Barrero, 2007; Rodríguez y García, 2003).

Así, se puede enfocar el punto esencial del aprendizaje organizacional como el desarrollo de la capacidad de aprendizaje de sus integrantes, y sumado a ello, la posibilidad de pensar en las maneras de fomentar las vías para desarrollar esta capacidad; este proceso de aprendizaje permanente en el contexto laboral, no supone una postura individual, limitada a la interrelación del profesional o del trabajador con su área de trabajo, sino que implica el vínculo con quienes comparte este espacio laboral, pues el aprendizaje, por su carácter social, es estimulado y desarrollado en el trabajo en grupos, en el intercambio de ideas valiosas, en la crítica oportuna o en la sugerencia pertinente, en la colaboración para generar ideas nuevas o indagar acerca de nuevos procedimientos que permitan la utilización cooperativa de ese conocimiento (Stable, 2011).

Lograr un cambio dirigido y planificado que permita la adaptación de los integrantes a la organización es uno de los grandes objetivos del aprendizaje organizacional, y trae como beneficios no solo la rápida inserción laboral de nuevos integrantes, sino también analizar y 
mejorar las prácticas laborales efectivas, potenciar las funciones de quienes se desempeñan en la institución y readecuar los medios usados para el logro de los objetivos y metas.

Cuando una organización identifica de manera rápida y eficiente sus estrategias de éxito frente a los requerimientos del medio, mantiene una ventaja competitiva sobre otras organizaciones; para ello, debe tener claridad de cuáles son esas estrategias y debe aprender de modo consciente y cooperativo (Barquero y Montero, 2013; Tovar, 2008). En las organizaciones, el aprendizaje de grupo tiene tres dimensiones críticas: la necesidad de pensar profundamente sobre los problemas más complejos, la necesidad de una acción coordinada e innovadora y finalmente, la presencia y responsabilidad de los miembros del grupo en otros grupos (García y Dutschke, 2007). De esta manera, el aprendizaje organizacional es una herramienta de cambio frente a una realidad social dinámica e inestable, a la que la organización debe adaptarse.

La importancia del aprendizaje metacognitivo para la organización es la capacidad de generar conciencia sobre las respuestas de ésta al medio, así como también, sobre las estrategias que ha utilizado para esa tarea. En este sentido, es una herramienta que permite obtener un conocimiento de la organización y sus acciones, que otorga una base segura de efectividad frente a las decisiones que se están ejecutando, lo cual es conocido y compartido por sus integrantes, permitiendo el uso de estrategias para mantener un control cooperativo y estratégico en función de las decisiones (Stable, 2011; Schechter y Qadach, 2014).

La organización escolar, al decir de Gairín y Goikoetxea (2008), aprende cuando la ejecución de las tareas que sus miembros realizan, individual o colectivamente, mejora constantemente, ya sea porque los procedimientos internos se mejoran y/o porque la interrelación entre los objetivos, los recursos y el sistema relacional se hace, en el nivel organizativo, menos disfuncional. Es decir, una organización que aprende es aquella que proporciona distintas opciones para que las personas se desarrollen en el quehacer y en el logro de las metas institucionales.

La escuela como organización, además de ser un contexto privilegiado para el aprendizaje escolar, también puede aprender y generar conocimiento sobre sí misma. Una escuela aprende cuando, por ejemplo, piensa, desarrolla y evalúa acciones que mejoran la convivencia y el clima del aula; cuando por acciones deliberadas y planificadas logra sostener a sus alumnos dentro sin resignar la enseñanza y el aprendizaje; es decir:

...cuando revierte gracias a la intervención de sus miembros, circuitos negativos de calidad; cuando sus profesores sienten que aprenden en las instancias de reflexión institucional; cuando pueden recuperar de su historia pasada alternativas de trabajo que resultaron valiosas; cuando puede deshacerse de las que ya no resultan eficaces; cuando a sus profesores les interesa aprender y compartir lo que aprenden. (Romero, 2007, p. 21)

En esta perspectiva, se puede entender que este aprendizaje es relevante para la gestión escolar, en el sentido que aporta un proceso de control y revisión acerca de las determinaciones 
de planificación y uso de los recursos de la organización escolar. La gestión escolar encierra en sí grandes desafíos que provienen del espacio y el tiempo en que se desarrolla, produciendo, en consecuencia, nuevos conocimientos sobre sí misma como proceso continuo. Las instituciones escolares poseen variadas herramientas de gestión que facilitarían el aprendizaje organizacional en la medida que generen verdaderos y efectivos procesos de participación.

Dado que la escuela es un escenario donde confluyen diversos actores, que generan tanto experiencias de aprendizaje individual como colectivo, esta debería constituirse en un espacio fértil de generación de aprendizajes. Sin embargo, muchas de esas experiencias no son aquilatadas en forma adecuada ni suficiente, debido a que las acciones y proyectos que se desarrollan no tienen incorporado el componente investigativo y de sistematización de estos, lo que implicaría una menor capacidad de enfrentar los procesos de cambio que le demanda el medio; una escuela que aprende, tiene a su favor, la capacidad de tener en cuenta un conjunto de informaciones que permiten contextualizar las demandas del medio, y por tanto, adecuar las decisiones y cambios que puede generar la escuela de modo de lograr lo que Gairín y Goikoetxea (2008) denominan como adaptación eficiente a su contexto.

En este proceso, que la institución genere estos procesos de aprendizaje en una modalidad metacognitiva, permitiría que las informaciones y decisiones que se deben tomar en cuenta, fueran más certeras y participativas, permitiendo un proceso de aprendizaje más pertinente, que si solo se generaran a partir de los mecanismos de registro o de investigación tradicionales, las cuales no son tan participativas, aspecto que es valorado por los integrantes de la comunidad educativa como característica de una gestión efectiva (Barquero y Montero, 2013).

Para finalizar este apartado, es necesario señalar que la metacognición es una herramienta fundamental del aprendizaje del siglo XXI, puesto que faculta a la persona para evaluar la información que le llega desde el medio, así como las conductas que decide llevar a cabo en relación con esa información; permite, a la vez, conocer y modificar. De este modo, aplicada al aprendizaje organizacional, será una herramienta que permita a la institución escolar, aprender de sí misma y buscar los caminos más óptimos para generar cambios.

\section{Metacognición institucional e innovación en la gestión escolar}

Arístegui, Bazán, Leiva, López, Muñoz y Ruz (2005) proponen el concepto de Metacognición Institucional (MI), señalando que es un proceso que se gesta cuando una organización completa actúa como una unidad o un conjunto para desarrollar, deliberadamente, procesos de autoconocimiento y autorregulación con el objeto de mejorar sus prácticas. Es decir, cuando en forma consciente, planificada y participativamente asume procesos de intercambio cuyo propósito es una mejor comprensión de sus formas de interacción, sus conflictos, dificultades y potenciales. 
Esta capacidad de autoconciencia de la institución debe sustentarse además, en un proceso de diálogo participativo, el cual permita canalizar la información de los aspectos que deben ser modificados; esto es relevante considerando que, en algunas situaciones, se observa una falta de procedimientos de las instituciones educacionales para rescatar la voz de los grupos minoritarios o excluidos. Con esta herramienta, se pueden tener espacios institucionalizados para recogerla y, de esta forma, lograr su pleno reconocimiento de la institución. Dicho de otro modo, es la capacidad de generar conciencia sobre los procesos de la institución y hacerlos emerger como factores de cambio, dando, de manera natural, un espacio de participación y valoración a todos los integrantes de la comunidad.

La MI mostraría varios beneficios: permite organizar responsabilidades de una manera clara y equilibrada, aumentando el logro de los procesos metacognitivos de los individuos; así mismo, fortalecer el desarrollo de estrategias de apoyo entre los integrantes del grupo o comunidad a través de la identificación de limitaciones en el desarrollo de tareas; coordinar esfuerzos entre los integrantes de la comunidad educativa, aumentando el logro de metas y de motivación; generar espacios de aprendizaje más solidarios, colaborativos y democráticos (Arístegui et al., 2005).

Así, la Ml surge como una herramienta de desarrollo organizacional relacionada con los procesos de aprendizaje de la organización, investigación y participación, que permitirían fortalecer la gestión educativa. La MI se produce en las diversas interacciones, en conversaciones claves para la acción y en la coordinación de esas acciones en el contexto institucional de la escuela, donde el equipo que dirige la institución es el actor clave y para ello, debe generar confianza con todos los actores (Rojas y Gaspar, 2006).

Por lo anteriormente señalado, se ve muy relacionada con el ámbito de la gestión educativa, la cual debiera ser de tipo participativa, y considerar las opiniones y necesidades de todos los integrantes del establecimiento, de manera que se logre una comunicación fluida y eficaz frente al manejo de informaciones y la toma de decisiones (Abarca, Aguilar, Cambronero, Chavarría, Moya y Murillo, 2013). Otro recurso de relevancia para lograr una gestión educativa efectiva, es el trabajo colaborativo entre docentes, así como entre docentes y directivos, lo cual permitiría el desarrollo de mayores instancias de participación, fomentando la generación, implementación y evaluación de proyectos educativos institucionales (o de centros) de manera democrática.

En esta perspectiva, un procedimiento fundamental es el proyecto educativo del establecimiento (centro), pues es considerado un instrumento válido en el mejoramiento de la calidad de la educación al dar las orientaciones que se proponen desde la comunidad educativa. Por ello, su construcción debe ser participativa, comprometiendo la voluntad y la adhesión progresiva de todos los actores de la comunidad educativa. Este tipo de experiencia de aprendizaje colectivo permite desarrollar una cultura organizacional participativa y comprometida con el desarrollo institucional. 
La Ml como herramienta permitiría el monitoreo participativo de las situaciones positivas y negativas en el establecimiento, lo que ayudaría a obtener información relevante y contextualizada de las necesidades de la comunidad escolar para la toma de decisiones y la asignación de funciones. Además, permitiría generar espacios de discusión para elaborar colaborativamente acuerdos y decisiones, lo cual redunda en que la gestión de la institución escolar se caracterice como democrática.

Esta manera de entender la Ml aplicada a la gestión escolar, como instrumento que permite conocer realidades del centro, a partir de una reflexión compartida, es lo que fundamenta la idea de que esta herramienta es un modo innovador de gestión del conocimiento, el cual sería, mejor dicho, una gestión del aprendizaje de la organización escolar, pues el foco está en el cambio consciente y efectivo de la institución. Ello, por cuanto busca generar acuerdos entre los integrantes de la comunidad educativa, que permitan mejorar o potenciar las realidades que se han reconocido en el proceso de reflexión cooperativa.

\section{Conclusiones}

La metacognición institucional como herramienta para el aprendizaje organizacional, cobra mucha importancia en las instituciones educacionales en la medida que se constituye en garantía para el logro de la calidad de la educación. Una organización que aprende de manera inductiva de sus procesos, genera conocimiento propio desde la participación y la reflexión colectiva, permitiendo un cambio con sentido y pertenencia a la realidad de la comunidad educativa.

Para ello, debe ser liderada por directivos visionarios, estratégicos, confiables y con capacidad para generar las conversaciones para coordinaciones pertinentes y oportunas. Desde esta perspectiva, las funciones de aprendizaje no deben entenderse como propias de un sistema consciente, dialógico y participativo (Arístegui et al., 2005; Barquero y Montero, 2013).

Sin embargo, el conocimiento desarrollado en las instituciones educativas habitualmente no es sistematizado, generando una pérdida de energía y de oportunidad para producir conocimiento propio desde la práctica. Del mismo modo, en el ámbito curricular, las organizaciones escolares en general tienen a su haber muchas experiencias en el desarrollo de proyectos propios o establecidos externamente, implementación de diversos programas que provienen de las políticas públicas y de la ejecución de propuestas relacionadas con diferentes ámbitos del devenir escolar, los que permiten generar algunos resultados para esas políticas, pero no necesariamente para las necesidades de la comunidad educativa.

La metacognición institucional, desde esta perspectiva, aporta a que las diversas estrategias (de gestión y/o investigativas) y acciones desarrolladas en el contexto escolar, se sostengan en la sistematización del aprendizaje logrado por medio del seguimiento y monitoreo oportuno. Lo anterior, se podría observar en el desarrollo de momentos de reflexión pedagógica 
en los docentes, actividades de investigación, acción en la escuela, sesiones de análisis de las situaciones de convivencia escolar, actividades de evaluación de la participación en actividades de la escuela, entre otras.

De esta forma, la realización de una gestión basada en la reflexión metacognitiva, permite hacer más consciente los aprendizajes construidos en el contexto de los procesos de gestión escolar, para los miembros de las comunidades educativas. Asimismo, estimula la participación efectiva y consciente de los diversos actores generando climas y culturas escolares más constructivas.

Finalmente, es relevante señalar que esta herramienta plantea una innovación a la gestión escolar en tres ámbitos. En primer lugar, el cambio de la dimensión del manejo de información de la organización, pasando de la mera clasificación de indicadores o recolección de datos, a la sistematización significativa para el cambio educativo. En segundo lugar, sería una innovación de la forma en que habitualmente se toman las decisiones, permitiendo un empoderamiento de los distintos actores de la comunidad educativa, y a través de ello, de las propuestas de formación democrática en las escuelas. Finalmente, en tercer lugar, es una innovación respecto a cómo se entiende el aprendizaje organizacional, enmarcándolo en la perspectiva teórica del constructivismo, donde el aprendizaje de cualquier organismo (u organización) se basa en la posibilidad de cambiar su destino y posibilidades, a partir de la interacción social y el uso del razonamiento.

\section{Referencias}

Abarca, Y., Aguilar, L., Cambronero, M., Chavarría, J., Moya, L., y Murillo, J. (2013). La gestión de la institución educativa y su vínculo con la comunidad. Revista Gestión de la Educación, 3(1), 83124. Recuperado de: $\underline{\text { http://revistas.ucr.ac.cr/index.php/gestedu/article/view/10638/10035 }}$

Akyol, Z. y Garrison, D. R. (2011). Assessing metacognition in an online community of inquiry. Internet and higher education, 14(3), 183-190. Recuperado de: http://www.sciencedirect. com/science/article/pii/S1096751611000066

Arístegui, R., Bazán, D., Leiva, J., López, R., Muñoz, B. y Ruz, J. (2005). Hacia una pedagogía de la convivencia. Psykhe, 14(1), 137-150. Recuperado de: http://www.scielo.cl/scielo. php?script= sci arttext\&pid=S0718-22282005000100011\&lng=es\&tlng=es. $\quad 10.4067 /$ $\underline{\mathrm{S} 0718-22282005000100011}$

Añino, M. y Perassi, M. (2008). Evaluación formativa y metacognición. Una experiencia innovadora en un curso de Bioingeniería. (VI Congreso Argentino de Enseñanza de la Ingeniería CAEDI). Recuperado de: http://www.caedi.org.ar/pcdi/PaginaTrabajosPorTitulo/7-598.PDF 
Arancibia, V., Herrera, P. y Strasser, V. (2008). Manual de psicología educacional. Santiago de Chile: Ediciones Universidad Católica.

Barquero, M. A. y Montero, M. (2013). Análisis comparativo de la gestión educativa como agente de cambio e innovación ante los complejos ambientes educativos, caso de las organizaciones: Colegio Nuevo Mundo y Colegio Técnico Profesional Piedades Sur, San Ramón. Revista Gestión de la Educación, 3 (2), 43-67. Recuperado de: http://revistas.ucr. ac.cr/index.php/gestedu/article/view/10648/10044

Barrero G., N. (2007). Aprendizaje metacognitivo de competencias profesionales. Educación XXI, 10, 39-60. Recuperado de: http://revistas.uned.es/index.php/educacionXX1/article/view/296

Briñol, P. y De Marree, K. (2012). Social metacognition. New York: Psichology Press.

Chin, M. M., y Kuo, S. W. (2010). From metacognition to social metacognition: similarities, differences, and learning. Journal of Education Research, 3(4), 321 - 338. Recuperado de: http://eds.b.ebscohost.com/eds/detail/detail?sid=d74c427f-5d83-4065-b6af-9ccb1a4d1

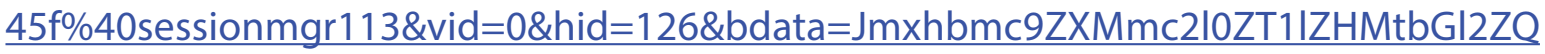
$\% 3 d \% 3 d \# A N=58057162 \& d b=e h h$

Coll, C., Palacios, J. y Marchesi, A. (comps.). (2001). Desarrollo psicológico y educación. Psicología de la Educación Escolar (Vol.II). Madrid: Alianza.

Collazos, C., y Mendoza, J. (2006). Cómo aprovechar el aprendizaje colaborativo en el aula. Educación y Educadores, 9(2), 61-76. Recuperado de: http://www.redalyc.org/articulo. $\underline{\text { oa?id }=83490204}$

Gairín, J.y Goikoetxea, J. (2008). La investigación en organización escolar. Revista de Psicodidáctica, 13(2), 73-95. Recuperado de: http://www.redalyc.org/articulo.oa?id=17513206

García, J. y Dutschke, G. (2007). Las organizaciones con capacidad de aprendizaje: A propósito de una revisión de la literatura. ACIMED [online], 16(5). Recuperado de: http://scielo.sld.cu/ scielo.php?script=sci arttext\&pid=S1024-4352007001100005\&lng=es

Larkin, S. (2009). Socially mediated metacognition and learning to write. Thinking Skills and Creativity, 4, 149-159. Recuperado de: http://www.sciencedirect.com/science/article/pii/ $\underline{\mathrm{S} 1871187109000479}$ 
Martínez F., J. (2004). Concepción de aprendizaje, metacognición y cambio conceptual en estudiantes universitarios de psicología. (Tesis de Doctorado). Universidad de Barcelona. Recuperado de: www.um.es/analesps/v23/v23 1/02-23 1.pdf

Mateos, M. (2001). Metacognición y educación. Buenos Aires: Aique.

Pozo, J. (2001). Estrategias de aprendizaje. En: Coll, C., Palacios, J. y Marchesi, A. (Comps.). Desarrollo psicológico y educación. Psicología de la Educación. Madrid: Alianza.

Rodríguez, M. y García, I. (2003). Formación de competencias para la autorregulación del aprendizaje en la empresa.(IVCongreso Latinoamericano de Sociología delTrabajo). Recuperado de:http://biblioteca. clacso.edu.ar/ar/libros/cuba/cips/caudales05/Caudales/ARTICULOS/ArticulosPDF/0525GR103.pdf

Rojas, A. y Gaspar, F. (2006). Bases del liderazgo en educación. Colección: Lideres escolares, un tesoro para la educación. Santiago de Chile: OREALC/UNESCO.

Romero, C. (2007). Laescuela media en la sociedad del conocimiento. Ideasy herramientas para la gestión educativa. Autoevaluación y planes de mejora. Buenos Aires: Ediciones Novedades Educativas.

Schechter, C. y Qadach, M. (2014). Toward an organizational model of change in elementary schools: The contribution of organizational learning mechanisms. Educational Administration Quarterly, 50, 577-609. Recuperado de: http://eaq.sagepub.com/content/48/1/116.abstract

Stable, Y. (2011). Modelo de aprendizaje organizacional para organizaciones de información. ACIMED, 22(3), 237-250. Recuperado de: http://scielo.sld.cu/scielo.php?script=sci arttext\&pid=S1024-4352011000300005\&lng=es

Sandi-Urena, S., Cooper, M., y Stevens, R. (2011). Enhancement of metacognition use and awareness by means of a collaborative intervention. International journal of science education, 33(3), 323-340. Recuperado de: http://www.tandfonline.com/doi/ abs/10.1080/09500690903452922

Tovar, J.C. (2008). Modelo metacognitivo como integrador de estrategias de enseñanza yestrategias de aprendizaje de las ciencias, y su relación con las competencias. Revista lberoamericana de Educación, 46(7). Recuperado de: http://www.rieoei.org/deloslectores/2161Tovarv2.pdf

Zorzoli, P., Lopardo, G. y Pérez, A. (1999). Triadas para la intervención solidaria: Una metodología didáctica. Gestión ambiental, 5, 35-49. Recuperado de: http://ceachile.cl/revista/ ARCHIVOS\%20PDF/GA\%205\%20Zorzoli\%20et\%20al.\%201999.pdf 